\title{
INTELIGENCIA EMOCIONAL E INICIATIVA EMPRENDEDORA: FUTUROS EMPRENDEDORES EN LA FORMACIÓN PROFESIONAL
}

\author{
Noemí Castilla Gutiérrez \\ I.E.S La Orden \\ Ncastillagutierrez@gmail.com
}

Recepción Artículo: 27 octubre 2021 Admisión Evaluación: 27 octubre 2021 Informe Evaluador 1: 28 octubre 2021 Informe Evaluador 2: 29 octubre 2021

Aprobación Publicación: 30 octubre 2021

\section{RESUMEN}

Actualmente en las empresas, tanto para los equipos líderes o directivos como para los propios trabajadores en su conjunto, se empieza a trabajar con estrategias de inteligencia emocional, con el objetivo de mejorar el clima y el rendimiento laboral. Competencias tales como la autoconciencia o Mindfulness, autogestión de las emociones, confianza en uno mismo, empatía, orientación hacia el logro e iniciativa emprendedora, junto con habilidades sociales indispensables para su propia conexión con el entorno, son las bases del éxito de un equipo de trabajo. Basándonos en esta nueva tendencia del desarrollo de soft skills en las empresas, no podemos olvidar que la educación es la base para la adaptación del individuo a la nueva sociedad y a las últimas tendencias sociales y para la inserción laboral del mismo atendiendo a los nuevos entornos laborales.

Es por ello, que, teniendo en cuenta que en la etapa de la Formación Profesional, independientemente de la familia formativa, se educa para la inserción laboral tanto por cuenta ajena (módulo de Formación y Orientación Laboral) como por cuenta propia (módulo de Empresa e Iniciativa Emprendedora), debemos formar a docentes capaces de fomentar y trabajar la inteligencia emocional de nuestro alumnado, capacitándolos con las soft skills necesarias para ser jóvenes con iniciativa emprendedora tanto para la creación de nuevas formas de negocios y de emprendimiento, como para ser futuros intraemprendedores y trabajadores de éxito para las organizaciones, para la sociedad y para ellos mismos como personas.

Palabras claves: inteligencia emocional; iniciativa emprendedora; formación profesional; soft skills; psicología y educación

\section{ABSTRACT}

Emotional intelligence and entrepreneurship: future entrepreneurs in vocational training. Currently, companies are working with emotional intelligence strategies for the leading or managerial teams and for the workers in general, with the aim of improving the climate and work performance in the work environment. 


\section{INTELIGENCIA EMOCIONAL E INICIATIVA EMPRENDEDORA: FUTUROS EMPRENDEDORES EN LA FORMACIÓN PROFESIONAL}

Competences such as self-awareness or Mindfulness, self-management of emotions, self-confidence, empathy, orientation towards achievement, entrepreneurial initiative and essential social skills for their own connection with the environment, are the bases of the success of a work team. Taking into account this new trend in the development of soft skills in companies, it must not be forgotten that education is the basis for the adaptation of the individual to the new society and the latest social trends and for the job insertion with the new work environments.

That is why, taking into account that in the Vocational Training stage, regardless of the training family, students are educated for job insertion both as an employee (Training and Labor Orientation module) and on their own (Company and Entrepreneurial Initiative module), we must train teachers capable of promoting and working on the emotional intelligence of our students, training them with the soft skills necessary to be young people with entrepreneurial initiative both for the creation of new forms of business and entrepreneurship, as well as to be future successful intrapreneurs and workers for organizations, for society and for themselves as individuals.

Keywords: emotional intelligence; entrepreneurship; professional training; soft skills; psychology and education

\section{INTRODUCCIÓN}

El trabajo que vamos a desarrollar a continuación, parte de la siguiente afirmación:

En la actualidad no sólo se nos juzga por lo más o menos inteligentes que podamos ser, ni por nuestra formación o experiencia, sino también por el modo en que nos relacionamos con nosotros mismos y con los demás, es decir, se buscan "Talentos Humanos" con "Inteligencia Emocional" (Coleman y Cherniss, 2013)

A partir de este planteamiento y nuevas tendencias, este trabajo pone el énfasis en la importancia de la intervención psicológica y educativa para potenciar la inteligencia emocional del alumnado y su iniciativa activa y emprendedora.

La idea nace de la oportunidad que se brinda en la Formación Profesional con los módulos de inserción laboral directa (módulo de Formación y Orientación Laboral) y los módulos que potencian la iniciativa emprendedora para la futura creación de nuevas empresas y nuevos estilos de profesionales más innovadores (módulo de Empresa e Iniciativa Emprendedora). Esta oportunidad pretende crear agentes activos en una sociedad en constante cambio y en una actualidad tecnológica, innovadora y que exige cualidades personales mucho más fuertes para la creación de grandes equipos de trabajo. El Talento Humano y la Inteligencia Emocional son la base para crear este nuevo perfil activo en la sociedad.

Se describirán cuáles fueron y siguen siendo los objetivos iniciales, qué herramientas se utilizan y cómo pueden servir para motivar y orientar a otros docentes, y cuáles son los primeros resultados obtenidos, en comparación con métodos más tradicionales de educación.

\section{OBJETIVOS DE LA INVESTIGACIÓN}

Partiendo de los objetivos básicos de la educación, establecidos en las últimas legislaciones que regulan la enseñanza en nuestro país, y en Europa en general, pero concretados actualmente en la Ley Orgánica 3/2020, de 29 de diciembre, por la que se modifica la Ley Orgánica 2/2006, de 3 de mayo, de Educación, se establece que:

La enseñanza básica persigue un doble objetivo de formación personal y de socialización, integrando de forma equilibrada todas las dimensiones. Debe procurar al alumnado los conocimientos y competencias indispensables para su desarrollo personal, para resolver situaciones y problemas de los distintos ámbitos de la vida, crear nuevas oportunidades de mejora, así como para desarrollar su socialización, lograr la continuidad de su itinerario formativo e insertarse y participar activamente en la sociedad en la que vivirán y en el cuidado del entorno natural y del planeta.». (Art.4)

Es por ello, que el presente trabajo tiene como objetivos:

Desarrollo personal y social del alumnado

Potenciar habilidades e inteligencia emocional para despertar la iniciativa emprendedora del alumnado 
Aportar herramientas para el profesorado, como guías o formadores de personas que pueden cambiar y mejorar el mundo.

Participar activamente en los avances de la sociedad.

\section{PARTICIPANTES}

Los participantes con los que se ha trabajado y se está trabajando para la consecución de los objetivos planteados, son el alumnado de Formación Profesional, tanto Grado Medio como Grado Superior, especialmente desde los módulos transversales de Formación y Orientación Laboral (1ํㅜㄴ curo) y de Empresa e Iniciativa Emprendedora (2ํㅡㄴ curso).

Se trabaja con este alumnado puesto que están formándose específicamente en una profesión, y tienen como objetivo principal la inserción laboral o la creación de nuevos empleos en la sociedad, ya que se consideran agentes activos para el desarrollo social y económico del país.

\section{METODOLOGÍA E INSTRUMENTOS UTILIZADOS}

La docencia debe partir de la base de que somos profesionales cuya principal función es guiar al alumnado para la consecución de las competencias, claves para el desarrollo de la inteligencia emocional, despertando así el talento interno de cada uno y la motivación para la creatividad y la participación activa por el desarrollo y el crecimiento.

Se trabaja desde una metodología en positivo, donde se refuerza los logros conseguidos y se motiva ante posibles errores o despistes del alumnado. Se guía a través de las actividades, pero no se exige la intervención del alumnado, se busca la iniciativa y la participación voluntaria en todo momento, con refuerzos positivos.

"Los procesos de enseñanza-aprendizaje y sus metodologías didácticas buscan conseguir actitudes abiertas, desde las que se acepten las reglas mínimas humanas -pacíficas y de comprensión mutua- hasta la disposición al comportamiento ético" (Llanes Ordónez y Rodríguez Moreno, 2019)

Las competencias a trabajar serán principalmente:

Conciencia de uno mismo: Partir del autoconocimiento supone saber sacar de nosotros mismos nuestro verdadero potencial y nuestras carencias, es decir, ser capaces de auto-observarnos, creer en nosotros y ser autocríticos en busca de la mejora. Teniendo en cuenta que una de las principales características del emprendedor es la autoconfianza y la autocrítica con el deseo de mejorar y evolucionar, los programas de orientación enfocados al conocimiento de uno mismo, son los más adecuados para que el alumnado de los ciclos formativos empiece a conocerse y a creer en sí mismo, y así poder llegar a tomar la decisión de arriesgarse y adentrarse en la iniciativa emprendedora.

Autogestión. La organización y planificación son la base de cualquier iniciativa laboral o emprendedora, y es la única forma de asegurar el éxito en nuestra participación activa en la sociedad.

Conciencia social. Es importante aterrizar en la realidad de lo que pasa en nuestro entorno, cuáles son las exigencias, cuáles son las necesidades y qué podremos aportar para participar y mejorar el mundo que nos rodea.

Habilidades sociales. No se trata de ser camaleones sociales y adaptarnos a lo que quieren otras personas, sino de ser capaces de reconocernos a nosotros mismos, reconocer a los demás y ser empáticos, asertivos y con capacidad de liderazgo para ser motores o motivadores en la activación y el desarrollo positivo social.

Los instrumentos utilizados para cada una de estas competencias o soft skills han sido:

Conciencia de uno mismo:

Ejercicios de Mindfulness

Ejercicios de reconocimiento de cualidades positivas y a mejorar de uno mismo, y de reconocimiento de cualidades positivas del compañero.

Test estandarizados

Exposición al grupo con reconocimiento de sensaciones. 


\section{Autogestión}

Propuestas propias de mejora, en función al análisis de los ejercicios de autoconocimiento.

Agenda de trabajo y planteamiento organizado de objetivos con respecto a la búsqueda de trabajo y a la creación de una idea de negocio innovadora

Análisis de plazos y consecución de objetivos de agenda

Planteamientos de mejora y nuevos objetivos y plazos de consecución.

Conciencia Social

Estudio y análisis del mundo laboral y empresarial. ¿Qué pasa en el mundo? ¿Cuáles son las últimas tendencias?

Autoanálisis sobre mis posibles aportaciones a la sociedad.

Participación social activa: ofertas de trabajos accesibles e ideas de negocios viables.

Valores y Responsabilidad Social: Cómo puedo mejorar y ayudar ante los problemas sociales. Ejercicios prácticos y viables.

Habilidades Sociales

Empatía. Consiste en aprender a ponerse en el lugar de otras personas, conectar con sus sentimientos y con su forma de ver y vivir las situaciones. Ejercicios de observación y anotación de actitudes y expresiones de otros (más allá de las palabras). Entrevistas a otras personas (escucha activa). Adivina qué siente (ejercicios grupales)

Asertividad. Se trata de aprender a defender nuestras necesidades y derechos sin faltar el respeto a los demás ni a uno mismo. Se realizan ejercicios de Teatro, Roll-Play, Scape Room.

Inteligencia Emocional. Capacidad que todos tenemos o podemos desarrolla para manejar de forma eficaz las emociones y generar, a partir de ellas, motivaciones beneficiosas para la persona. Se trabajará con diarios de emociones, ejercicios de escuchas activas entre el alumnado (verbal y no verbal), rueda de la vida y charlas motivacionales de coaching (profesionales y emprendedores)

Liderazgo. Conseguir personas con capacidad de influir, motivar, organizar y guiar a otras personas de forma positiva y productiva. Ejercicios grupales de Roll-Play, Resolución Grupal de conflictos, Análisis de intervenciones grupales y de Simulación de Estilos de liderazgo.

\section{RESULTADOS ALCANZADOS}

Se trata de un trabajo que se ha desarrollado en los últimos cursos a través de los módulos mencionados con anterioridad (Formación y Orientación Laboral -FOL, y Empresa e Iniciativa Emprendedora- EIE).

Primeros resultados obtenidos en FOL:

Alumnado visiblemente más motivado e implicado en el aula. Incluso descartando la continuidad en otros módulos por razones motivacionales o personales, han seguido con entusiasmo el estudio de este módulo y con la idea de su utilidad en su futuro inmediato.

Inserción Laboral. Alumnado que han pasado pruebas de selección diversas y han encontrado su hueco en el mundo laboral tras la realización de las actividades de este módulo, reconociendo su mejora.

Primeros resultados obtenidos en EIE:

Alumnado con interés en el emprendimiento y con búsqueda activa de opciones para llevar a cabo sus ideas de negocio.

Alumnado visiblemente más motivado.

Aumento de la creatividad e interés por innovar y crear algo diferente que pueda ser útil en la sociedad.

\section{DISCUSIÓN}

Los resultados obtenidos se traducen finalmente en buenas calificaciones en las evaluaciones, buen clima en el ámbito laboral, profesorado más implicado, alumnado más motivado y una atmósfera educativa de creer en algo y en la posible participación activa e innovadora en la sociedad. 
Los objetivos de mejorar la Inteligencia Emocional del alumnado y la consecución del desarrollo del Talento Personal de cada uno, se han conseguido en la medida en la que se va viendo el despertar del interés y el aumento de la creatividad a lo largo del curso.

Estudios como los de Daniel Coleman y Cary Cherniss, sobre el trabajo de la Inteligencia Emocional en el mundo Laboral, son el referente de esta visión dentro educación, en el marco de la inserción laboral adecuada y actualizada.

Se trata de un trabajo en activo y con poco tiempo de experiencia, pues prácticamente, teniendo en cuenta las dificultades del curso 2019/20, ocasionadas por la pandemia, sólo se ha podido poner en marcha íntegramente en el curso 2020/21 y aún desarrollándose en este nuevo curso. Eso sí, cada año se enriquece el programa y se trabaja con mayor implicación, cuyos resultados empiezan a verse desde el primer momento en el alumnado (mayor interés y participación).

La idea es seguir trabajando en el aula, compartir experiencias con otros docentes y conseguir iniciativas emprendedoras para participar entre todos en el buen desarrollo y evolución de la sociedad.

\section{CONCLUSIONES}

Este trabajo pone de manifiesto la necesidad del trabajo desde la Psicología y la Educación en el marco de la Formación Profesional, para generar acciones positivas de intervención social, además del desarrollo personal del alumnado.

Formar y Orientar debe ser un proceso continuo desde que el alumnado entra en la comunidad educativa hasta que consigamos su inserción y participación activa en el mundo laboral y empresarial.

No debemos caer en la mera acción educativa con una calificación final, sino en la búsqueda de la intervención social y el bienestar futuro de nuestro alumnado y de nuestra sociedad.

Por todo ello, esta presentación pretende motivar el profesorado, aportar posibles herramientas y estructura a los módulos más directos de intervención en la sociedad, pero también para el resto del profesorado, ya que la comunidad educativa debe verse en conjunto y como un verdadero equipo de trabajo.

La Educación no cambia el mundo, cambia a las personas que van a cambiar el mundo

Paulo Freire

\section{REFERENCIAS BIBLIOGRÁFICAS}

Álvarez González, M.; y Bisquerra, R. (Coord.) (2005). Manual de Orientación y Tutoría. Praxis

Armando Corbin, J.(s.f.). 7 ejercicios y actividades para desarrollar tu Inteligencia Emocional. Piscología y Mente. https://psicologiaymente.com/psicologia/ejercicios-para-desarrollar-inteligencia-emocional

Bastida de Miguel, A.M. (2018, marzo). Sobre la Inteligencia Emocional. Psicología-Online.https://www.psicologia-online.com/sobre-la-inteligencia-emocional-2369.html

Cherniss, C. y Goleman, D. ((2013). Inteligencia Emocional en el trabajo. Editorial Kairós

Goleman, D. (2010). Inteligencia Emocional. Editorial Kairós

Hernández, C. (2020). Emprendedor en proceso: el enquiridión del emprendedor. Apple Books

Ley Orgánica 3/2020, de 29 de diciembre, por la que se modifica la Ley Orgánica 2/2006, de 3 de mayo, de Educación. (2020). Boletín Oficial del Estado, 340, sec. I, de 30 de diciembre de 2020, 122868 a 122953. https://www.boe.es/eli/es/lo/2020/12/29/3/dof/spa/pdf

Ministerio de Educación y Formación Profesional (s.f.) Recursos Profesorado FOL. Todofp. https://www.todofp.es/profesores/recursos/profesorado-fol.html

Muñoz Martínez, A. (s.f.). 10 Dinámicas de Liderazgo para Niños, Adolescentes y Adultos. Lifeder. https://www.lifeder.com/dinamicas-de-liderazgo/ 
Rodríguez Moreno, M.L.; Llanes Ordóñez, J. (2019) Formación y Orientación de Personas Adultas. Universo de Letras

Sanz Ponce, R. y Cárdenas Gutiérrez, A. (2021). ¿Cómo generar u sentimiento positivo hacia la competencia emprendedora en la escuela? Revista Andalucía Educativa. https://www.juntadeandalucia.es/educacion/portals/web/revista-andalucia-educativa/contenidos/-/novedades/detalle/8dKQZE7Sn2zC/como-generar-unsentimiento-positivo-hacia-la-competencia-emprendedora-en-la-escuela 